position for which no evidence is given, this research, however well intended, may be stigmatising in itself.

Cooksey, E. C. \& Brown, P. (1998) Spinning on its axes: DSM and the social construction of psychiatric diagnosis. International Journal of Health Services, 28, 525-554.

Crisp, A. H., Gelder, M. G., Rix, S., et al (2000) Stigmatisation of people with mental illnesses. British Journal of Psychiatry, 177, 4-7.

Foucault, M. (1967) Madness and Civilisation: A History of Insanity in the Age of Reason. London: Tavistock.

Levine, H. G. (1979) The discovery of addiction: changing conceptions of habitual drunkenness in America. Journal of Studies on Alcohol, 15, 493-506.

McMurran, M. (1994) The Psychology of Addiction. London: Taylor and Francis.

D. Heim Department of Psychology, The University of Strathclyde, Graham Hills Building, 40 George Street, Glasgow GI IQE

\section{Olanzapine and pancreatitis}

Two patients with chronic schizophrenia were admitted with acute pancreatitis. Olanzapine was used by both patients and may represent a further example of druginduced pancreatitis. Olanzapine is a thienobenzodiazepine similar in structure to clozapine; clozapine has been associated with the induction of acute pancreatitis (Frankenburg \& Kando, 1992; Gatto et al, 1998).

A 34-year-old man with chronic schizophrenia was admitted with abdominal pain. Two years previously he had had acute pancreatitis, but continued to drink alcohol (8 units/day). The patient was taking $20 \mathrm{mg}$ olanzapine daily. Glasgow criteria and computed tomography classified the episode as severe pancreatitis. The patient developed respiratory failure and required artificial ventilation for $\mathbf{1 1}$ days. A full recovery was made.

A 29-year-old man with chronic schizophrenia was admitted with abdominal pain. He had a past history of acute pancreatitis, having had an attack 8 years previously. The patient drank at least 15 units of alcohol per week. Regular medication was sulpiride and olanzapine $20 \mathrm{mg}$ daily. Diagnosis was made by computed tomography. The patient made an uncomplicated recovery. The same patient was readmitted 4 months later with another attack of acute pancreatitis - he had continued to take olanzapine and to drink alcohol.
Although both patients were regular consumers of alcohol, a known risk factor for the development of acute pancreatitis, we felt it unusual that two patients on olanzapine should present within such a short period of time. Clozapine has been associated with pancreatitis and has a similar structure to olanzapine; one might therefore suspect that olanzapine might cause pancreatitis. The evidence for olanzapine inducing pancreatitis is strengthened by the fact that in one of the patients a further episode occurred after rechallenge with the drug. It is possible that olanzapine acts in a synergistic fashion with alcohol in the pathogenesis of the disease. We propose that olanzapine should be used with caution with patients who drink alcohol on a regular basis or who have a previous history of pancreatitis.

Frankenburg, F. \& Kando, J. (1992) Eosinophilia, clozapine and pancreatitis. Lancet, 340, 251.

Gatto, E., Castronuovo, A. \& Uribe Roca, M. (1998) Clozapine and pancreatitis. Clinical Neuropharmacology, 2I, 203.

R. Hagger, C. Brown, P. Hurley Mayday University Hospital, London Road, Croydon, Surrey CR7 7YE

\section{Antidepressant augmentation with low-dose olanzapine in obsessive-compulsive disorder}

We read with interest the article on obsessive-compulsive disorder (OCD) and delusions, by O'Dwyer \& Mark (2000). The suggestion of a continuum of pathological beliefs in OCD from 'none' to 'delusional intensity' suggests the possibility of another continuum of mental disorders from OCD to psychosis.

The authors presented five cases of OCD associated with delusional beliefs. They concluded that these patients are best considered within an OCD management plan. Moreover, they do not recommend use of long-term antipsychotic medication since they consider that such patients are unlikely to respond. However, none of the five patients they reported upon was treated with low doses of an atypical antipsychotic in order to augment the action of serotoninspecific antidepressants, the preferred pharmacotherapy in OCD.

Recently, we have successfully treated a man in his 50 s who presented with a 7-year history of typical OCD. His problem was suitable for treatment using exposure and ritual prevention, combined with an antidepressant. He was referred for behavioural psychotherapy at the specialist unit based at the Maudsley Hospital. Although motivated to try this treatment he later found it too difficult to continue with this approach. He therefore remained under the care of his general practitioner (GP) who treated him with fluoxetine $20 \mathrm{mg}$ daily, on which he had only a very slight improvement in his symptoms. When we saw him at the request of his GP he was asking for relief from anxiety symptoms. Small doses of thioridazine produced unacceptable sideeffects, so olanzapine $2.5 \mathrm{mg}$ was substituted. After approximately 4 weeks his symptoms were almost completely gone, and at a follow-up appointment he stated that for the first time after 7 years of rituals and obsessions, he felt $90 \%$ better.

Initially, our choice of olanzapine was determined by the patient's need for anxiolysis. However, there is emerging evidence that olanzapine may augment the action of fluoxetine in the treatment of individuals with OCD (Weiss et al, 1999). Another possibility is that olanzapine was having a direct action on psychotic phenomena. Whatever the case, we would suggest that contrary to the recommendations for management offered by O'Dwyer \& Marks (2000) some treatment-resistant cases of OCD might respond to a therapeutic trial with low doses of an atypical antipsychotic in addition to a serotonin-specific antidepressant as usually recommended.

O'Dwyer, A. M. \& Marks, I. (2000) Obsessivecompulsive disorder and delusions revisited. British Journal of Psychiatry, 176, 28I-284.

Weiss, E. L., Potenza, M. N., McDougle, C. J., et a (1999) Olanzapine addition in obsessive-compulsive disorder refractory to selective serotonin reuptake inhibitors: an open-label case series. Journal of Clinical Psychiatry, 60, 524-527.

A. Marušič, A. Farmer SGDP Research Centre, Institute of Psychiatry, De Crespigny Park, London SE5 8AF

\section{Reboxetine-induced spontaneous ejaculation}

Delayed or absent ejaculation is a common side-effect of antidepressant drugs. Reboxetine, a selective noradrenaline reuptake inhibitor, is known for its lack of sexual side-effects. We report a case of reboxetineinduced spontaneous ejaculation. 
A 39-year-old married man was referred with recurrent depression. When euthymic his sexual function was normal. When depressed he had mildly decreased libido but normal erection, ejaculation and orgasm. He had poor response to, but no sexual side-effects with, fluoxetine $40 \mathrm{mg}$ for 8 weeks. Later his depression improved with reboxetine $4 \mathrm{mg}$ twice daily. However, he developed spontaneous ejaculations occurring 2-3 times a day. These were not preceded by sexual stimulation but were associated with pleasurable sensation. He also developed premature ejaculation during sexual intercourse. On discontinuing the medication, the spontaneous ejaculations and premature ejaculations subsided within 7 days.

This is the first report of reboxetineinduced spontaneous ejaculations. Seminal emission and ejaculation are controlled centrally by the anterior thalamic nuclei, preoptic nuclei and the median forebrain bundles, which are facilitated by dopamine and inhibited by $5-\mathrm{HT}_{1 \mathrm{~A}}$ antagonists and 5$\mathrm{HT}_{2}$ agonists. The spinal centres for emission, ejaculation and for bladder neck closure during ejaculation to prevent retrograde ejaculation are mediated by noradrenaline. Delayed, absent or retrograde ejaculation can be caused by sympatholytic antihypertensives, antipsychotics, selective serotonin reuptake inhibitors (SSRIs), surgical sympathectomy, spinal cord injury and diabetic autonomic neuropathy. The 5- $\mathrm{HT}_{1 \mathrm{~A}}$ agonist $8-\mathrm{OH}-\mathrm{DAPT}, 5-\mathrm{HT}_{2}$ antagonists nefazodone and adrenergic agents such as ephedrine, pseudoephedrine and phenylephrine can reduce ejaculatory latency and revert retrograde ejaculation to antegrade ejaculation (Wang et al, 1996;
Michael \& O'Keane, 2000). Reboxetine is a selective and potent inhibitor of noradrenaline reuptake and has little effects on other neurotransmitter systems. This could explain its relative lack of sexual side-effects. Reboxetine could have caused decreased ejaculatory latency as well as spontaneous ejaculations by reuptake inhibition and consequent increased activity of noradrenaline. Michael et al (1999) used the ability of nefazodone to cause spontaneous ejaculation by virtue of its $5-\mathrm{HT}_{2}$ antagonist effect to reverse SSRI-induced anorgasmia. Reboxetine could be a potential substitute for antidepressants which cause delayed or absent ejaculation/orgasm.

Michael, A. \& O'Keane, V. (2000) Sexual dysfunction in depression. Human Psychopharmacology, I5, 337-345.

— , Tubbe, P. A. \& Praseedom, A. (1999) Sertralineinduced anorgasmia reversed by nefazodone (letter). British Journal of Psychiatry, 175, 491.

Wang, R., Monga, M. \& Hellstorm, W. J. G. (1996) Ejaculatory dysfunction. In Male Infertility (ed. F. H. Comhaire), pp. 205-22I. London: Chapman \& Hall.

R. O'Flynn \& A. Michael Department of Psychiatry, West Suffolk Hospital, Bury St Edmunds IP33 2QZ

\section{A virtual cry for help?}

With the advent of the internet, and widespread publication of e-mail addresses on web pages, it is increasingly easy to correspond with members of the health professions. Such ease of access may be used to good effect, but is also open to abuse. For example, there is a report describing an individual who was saved after they published their intention of suicide on the internet. Furthermore, the Samaritans can be accessed by e-mail. Recently I received an e-mail purporting to be a suicide note. The mail apparently originated from an identifiable individual and their e-mail address was supplied. The letter expressed anger, with unambiguous suicidal intent, and contained some features suggestive of contact with mental health and social services. Having received the letter some 6 hours after it was sent I initially contacted the server who indicated that they could identify the subscriber and would be able to release this information to the police. I therefore contacted the local police and this information was passed to Scotland Yard who arranged a visit to the registered address. The police found the individual, whose signature was on the letter, safe and well. Apparently, at interview the individual, with the responsible adults at the address, denied sending the e-mail.

This e-mail could have been a genuine cry for help, which remains unmet, or the situation may have resolved. Alternatively, it may represent a hoax perpetrated by the individual concerned or another logging onto the server with, or without, permission. While appropriate use of e-mail is to be encouraged there is a great potential for abuse, with the concomitant waste of clinical and police time. As such letters will become more frequent it is essential that an appropriate response and procedure be established between the caring professions, police and internet service providers.

D. Ball The Oaks Resource Centre, 843 London Road, Thornton Heath, Croydon CR7 6JH

\section{One hundred years ago}

\section{Joint Counties Asylum, Carmarthen (Annual Report for 1899)}

... Dr. E. Goodall, the medical superintendent, points out in his report that "a thorough overhauling of the defective parts will be desirable before the building comes into use as a permanent accommodation."
Among the admissions is described a case of "moral insanity" - viz., a young girl admitted for the fourth time. "There being no defect or derangement of mind upon which a certificate of detention in an asylum could be returned she could not be kept here. This girl is a source of great trouble at home, but is neither mad enough to be in the asylum nor bad enough to be in gaol, and is probably best suited for a reformatory." ...

\section{REFERENCE}

Lancet, 13 October 1900, 1079.

Researched by Henry Rollin, Emeritus Consultan

Psychiatrist, Horton Hospital, Epsom, Surrey 